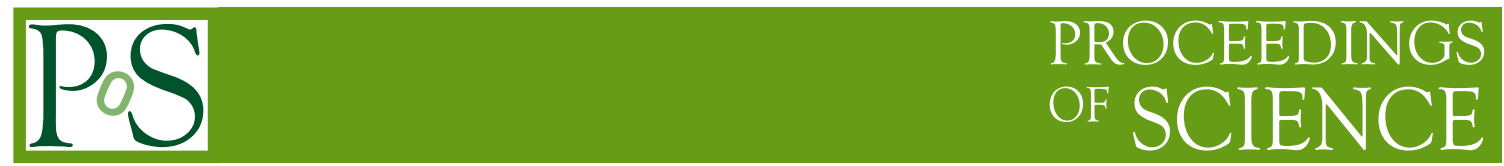

\title{
Highlights from the ATLAS experiment at the CERN LHC
}

I.I. Tsukerman on behalf of the ATLAS Collaboration* NRC KI "ITEP", Moscow, 117218, Russia

E-mail: ilya.tsukerman@cern.ch

Highlights from the ATLAS Experiment at CERN Large Hadron Collider are presented. Results shown are mostly based on the analysis of the complete 2015-2016 year dataset which corresponds to the integrated luminosity of $36 \mathrm{fb}^{-1}$ taken in $13 \mathrm{TeV}$ proton-proton collisions. Mainly recent measurements of Higgs boson production and decays are discussed. In addition, short summary of other results is also given.

Frontier Research in Astrophysics III (FRAPWS2018)

28 May - 2 June 2018

Mondello (Palermo), Italy

\footnotetext{
* Speaker.
} 


\section{Introduction}

\subsection{The LHC and its physics program}

The Large Hadron Collider (LHC) [1] at CERN provides proton-proton ( $p p$ ) collision at the center-of-mass energy $13 \mathrm{TeV}$ since 2015. Two huge multi-purpose experiments, the ATLAS [2] and CMS [3] as well as two big, but more dedicated experiments, the LHCb [4] and ALICE [5], operate at the LHC. In total, the ATLAS and CMS detectors each accumulated large amount of data corresponding to an integrated luminosity of more than $100 \mathrm{fb}^{-1}$ at $13 \mathrm{TeV}, 23 \mathrm{fb}^{-1}$ at $8 \mathrm{TeV}$ and $5 \mathrm{fb}^{-1}$ at $7 \mathrm{TeV} p$-collisions. The maximum instantaneous luminosity reached in 2018 is $2 \times 10^{34} \mathrm{~cm}^{-2} \mathrm{~s}^{-1}$ which exceeds the LHC design luminosity by a factor of two.

The wide-range physics program of the LHC includes:

- tests of the Standard Model of interactions (SM) in the new energy range;

- studies of properties of recently discovered Higgs boson $(h)$ with the mass $125 \mathrm{GeV}$ [6]-[7];

- direct and indirect searches for new physics beyond the Standard Model (BSM);

- studies of heavy ion collisions;

- heavy flavour physics;

- an extraction of the parton distribution functions (PDF) in the wide kinematic range as well as precision measurements of such important SM parameters as $m_{W}, \alpha_{S}, \sin ^{2} \theta_{W}$ etc.

Expected cross sections of different SM processes vary by many orders of magnitude from $100 \mathrm{mb}$ for the total $p p$ cross section to about $10-100 \mathrm{pb}$ for the SM Higgs boson production cross section. The LHC experiments are able to measure such cross sections with high precision.

\subsection{The ATLAS detector and physics}

The ATLAS detector [2] is a multipurpose particle physics apparatus with forward-backward symmetric cylindrical geometry. The inner tracking detector (ID) consists of a silicon pixel detector, a silicon microstrip detector, and a straw-tube transition radiation tracker. The ID is surrounded by a thin superconducting solenoid which provides a $2 \mathrm{~T}$ magnetic field, and by high-granularity liquid-argon (LAr) sampling electromagnetic (EM) calorimetry. The electromagnetic calorimeter is divided into a central barrel and end-cap regions on either end of the detector. In the region matched to the ID $\left(|\eta|<2.5^{1}\right)$, it is radially segmented into three layers. The first layer has a fine segmentation in $\eta$ to facilitate e $/ \gamma$ separation from $\pi^{0}$ and to improve the resolution of the shower position and direction measurements. In the region $|\eta|<1.8$, the electromagnetic calorimeter is preceded by a presampler detector to correct for upstream energy losses. An iron-scintillator/tile calorimeter gives hadronic coverage in the central rapidity range $(|\eta|<1.7)$, while a LAr hadronic

\footnotetext{
${ }^{1}$ ATLAS uses a right-handed coordinate system with the origin at the nominal interaction point (IP) in the centre of the detector, and the $z$-axis along the beam line. The $x$-axis points from the IP to the centre of the LHC ring, and the $y$-axis points upwards. Cylindrical coordinates $(r, \theta)$ are used in the transverse plane, $\theta$ being the azimuthal angle around the beam line. Observables labelled "transverse" are projected into the $x-y$ plane. The pseudorapidity is defined in terms of the polar angle $\theta$, as $\eta=-\ln \tan (\theta / 2)$.
} 
end-cap calorimeter provides coverage over $1.5<|\eta|<3.2$. The forward regions $(3.2<|\eta|<4.9)$ are instrumented with LAr calorimeters for both electromagnetic and hadronic measurements. The muon spectrometer (MS) surrounds the calorimeters and consists of three large air-core superconducting magnets providing a toroidal field, each with eight coils, a system of precision tracking chambers, and fast detectors for triggering. The combination of all these systems provides charged particle measurements together with efficient and precise lepton and photon measurements in the pseudorapidity range $|\eta|<2.5$. Jets and missing transverse energy, $E_{T}^{\text {miss }}$, are reconstructed using energy deposits over the full coverage of the calorimeters, $|\eta|<4.9$.

One should note that $p p$-collisions at $13 \mathrm{TeV}$ in the ATLAS and CMS interaction points occur with a frequency of $\approx 40 \mathrm{MHz}$, i.e. the interval between them is about $25 \mathrm{~ns}$. Thanks to high LHC luminosity and large proton-proton inelastic cross section, each collision of beam bunches results, in average, to more than thirty physics events simultaneously. This effect is usually called as "pileup" effect. Despite of such conditions, almost 95\% of events triggered in the ATLAS detector are good for physics analysis.

The ATLAS collaboration has a few working groups which concentrate on the following studies:

- SM (electroweak processes, single $W / Z$-production, photons, hadronic jets, soft processes);

- heavy flavours and light states (charmonia, bottomonia; rare decays of $B$-mesons etc);

- Higgs boson (decay channels, their combination, perspectives);

- top quarks (single and pair production; measurement of the mass);

- BSM searches in many final states;

- heavy ions (ion-ion and proton-ion interactions).

Results presented in this note are mostly based on the complete 2015-2016 dataset taken at $13 \mathrm{TeV}$ energy. It corresponds to the integrated luminosity $36 \mathrm{fb}^{-1}$. The summary of ATLAS results on cross section measurements of many SM processes is given in Fig.1(a). They are all in agreement with the most recent theoretical predictions, so we understand backgrounds to new physics. Further we concentrate mainly on the study of a Higgs boson production and its decays.

The note is organized as follows. Section 2 contains a brief summary of expected Higgs boson production cross sections in different mechanisms as well as branching ratios (BR) of its decay modes. In Section 3, the most sensitive bosonic decay modes of the SM Higgs boson $(h)$, namely the $h \rightarrow Z Z^{\star} \rightarrow 4 \ell, h \rightarrow \gamma \gamma$ and $h \rightarrow W W^{\star} \rightarrow \ell v \ell v$ are considered; Section 4 is devoted to the $h \rightarrow b \bar{b}$ decay mode and in Section 5 the $t \bar{t} h$ production is discussed. In Section 6 BSM searches of heavy scalar higgs boson, $H$, are summarized. Conclusions are drawn in Section 7 together with short-term plans.

\section{SM Higgs boson production and decays}

The Higgs boson in the SM provides all fundamental particles with masses. Before 2012 the $h$ boson mass was the last unknown parameter. From theoretical considerations (perturbative unitarity) $m_{h} \leq 1 \mathrm{TeV}$ is expected. The Higgs boson should have vacuum quantum numbers, i.e. zero 
spin and even parity $\left(J^{P}=0^{+}\right)$. In 2003, LEP experiments put the lower bound on its mass, $m_{H} \geq 114.4 \mathrm{GeV}$ at the $95 \%$ confidence level (CL); smaller masses were excluded at much higher CL [9]. Theoretical analyses of electroweak precision data predicted $m_{H}=94_{-24}^{+29} \mathrm{GeV}$, i.e. $m_{h} \leq 152 \mathrm{GeV}$ at the $95 \% \mathrm{CL}$ [10]. Indeed, a new boson with a mass of $125 \mathrm{GeV}$, i.e. in-between the mentioned limits, was discovered by the ATLAS [6] and CMS [7] Collaborations at the Large Hadron Collider (LHC) six years ago. ${ }^{2}$ This discovery was a great success of the SM as all measured properties of this particle were found to be compatible with the predictions for the $h$ boson.

A search for the SM Higgs boson at the LHC is a complicated task as expected signal production cross section is $\sigma_{h}=\mathrm{O}(10) \mathrm{pb}$ while background rates are much higher. Predicted $\sigma_{h}$ at $m_{h}=$ $125 \mathrm{GeV}$ rises with the center-of-mass $p p$ energy from $\approx 20 \mathrm{pb}$ at $8 \mathrm{TeV}$ to $\approx 50 \mathrm{pb}$ at $13 \mathrm{TeV}$ [12]. There are four main mechanisms of the SM Higgs boson production at the LHC energies: gluon fusion ( $\mathrm{ggF}$ ) via heavy-quark (mostly top) triangular loop, vector-boson fusion (VBF) where the Higgs boson is accompanied by two jets going at small polar angles, associated production with one vector boson $(V h$, i.e. together with $W$ or $Z$ ) and top-antitop fusion. The first and the last mechanisms as well as the second and the third ones have common couplings ( $t \bar{t} h$ and $V V h$, respectively). The expected $\sigma_{h}$ from these mechanisms are shown in Fig.1(b) as function of $m_{h}$ at $\sqrt{s}=$ $13 \mathrm{TeV}$ together with theoretical uncertainties [12]. It is seen that the ggF mechanism dominates while rates via $\operatorname{VBF}(V h, t \bar{t} h)$ mechanisms are ten (a few dozen) times smaller, respectively.

The expected branching ratios (BR) of the experimentally favourable decay modes are given in Table 1. Despite of 58\% probability, the $h \rightarrow b \bar{b}$ channel is very difficult experimentally due to a huge background and it is not possible to see it via the ggF mechanism. However, one can try to extract the signal in the associated production of the $h$ with a vector boson or a pair of top quarks. The $h \rightarrow W W^{\star} \rightarrow \ell v \ell v$ channel (here and further $\ell$ stands for an electron/positron or a muon) is better for searches, despite having branching ratio of only $\approx 1 \%$. However, it does not allow to reconstruct a Higgs boson mass. The cleanest decay channels where this is possible are the $h \rightarrow Z Z^{\star} \rightarrow 4 \ell\left(\mathrm{BR} \approx 1.3 \times 10^{-4}\right)$ and the $h \rightarrow \gamma \gamma\left(\mathrm{BR} \approx 2.3 \times 10^{-3}\right)$. In the last case a signal is searched above large background. Expected signal event rates normalized to $36 \mathrm{fb}^{1}$ data sample collected at $13 \mathrm{TeV}$ are also shown in Table 1 . Here 100\% detection efficiency is assumed.

\section{The $h \rightarrow \gamma \gamma, h \rightarrow Z Z^{\star} \rightarrow 4 \ell$ and $h \rightarrow W W^{\star} \rightarrow \ell v \ell v$ decay channels}

The brief summary of the ATLAS results on the $h$ boson measurements at $8 \mathrm{TeV}$ is given in Table 2. One can see that the Higgs boson signal strength is measured with $10-50 \%$ precision, depending on the decay mode and on the production mechanism. The spin and parity of the were determined to be equal to $0^{+}$. No deviation from the SM is found. Below we concentrate on the results obtained at $13 \mathrm{TeV}$.

\subsection{The $h \rightarrow Z Z^{\star} \rightarrow 4 \ell$ mode [20]}

The $h \rightarrow Z Z^{\star} \rightarrow 4 \ell$ signature is two pairs of isolated, opposite-sign leptons. The invariant-mass distribution, $m_{4 \ell}$, measured by the ATLAS experiment after the combination of all lepton cases [20]

\footnotetext{
${ }^{2}$ The CDF and D0 experiments at the FNAL Tevatron $p \bar{p}$-collider were able to find only an evidence for the Higgs boson production at $3 \sigma$ level [11].
} 


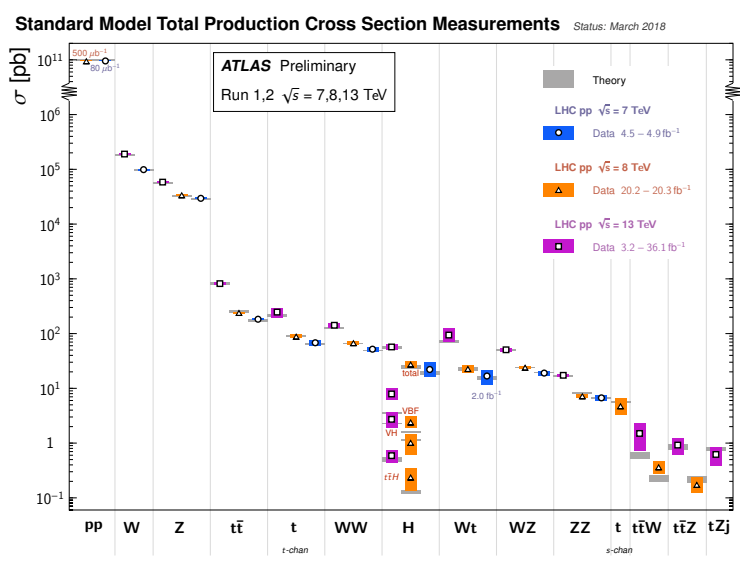

(a)

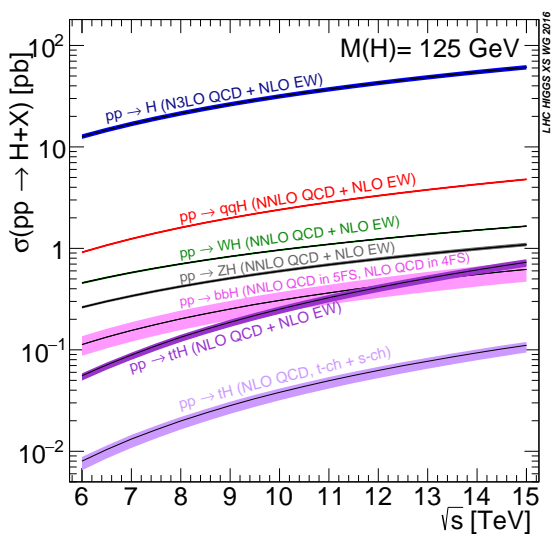

(b)

Figure 1: (a) A summary of measured cross sections of different SM processes in the ATLAS experiment [8]. (b) Predicted SM Higgs boson cross section at $m_{h}=125 \mathrm{GeV}$ in different production mechanisms as function of $\sqrt{s}[12]$.

\begin{tabular}{c|c|c|c}
\hline Decay mode & BR, $\%$ & Observability in the experiment & Event rates \\
\hline$h \rightarrow b \bar{b}$ & $57.5 \pm 1.9$ & Mainly in $V h$ and $t$ th production & $\geq 24000$ \\
$h \rightarrow W W^{\star}$ & $21.6 \pm 0.9$ & Leptonic decays of both $W$ & $\approx 17000$ \\
$h \rightarrow g g$ & $8.56 \pm 0.86$ & no good experimental signature & \\
$h \rightarrow \tau \tau$ & $6.30 \pm 0.36$ & Mainly in VBF production & $\approx 10000$ \\
$h \rightarrow c \bar{c}$ & $2.90 \pm 0.35$ & no good experimental signature & \\
$h \rightarrow Z Z^{\star}$ & $2.67 \pm 0.11$ & Leptonic decays of both $Z$ & $\approx 250$ \\
$h \rightarrow \gamma \gamma$ & $0.228 \pm 0.011$ & Big continuum background & $\approx 5000$ \\
$h \rightarrow Z \gamma$ & $0.155 \pm 0.014$ & Leptonic decays of $Z$ & $\approx 250$ \\
$h \rightarrow \mu \mu$ & $0.022 \pm 0.001$ & Big continuum background & $\approx 500$ \\
\hline
\end{tabular}

Table 1: Expected $h$ branching ratios at $m_{h}=125.1 \mathrm{GeV}$ as well as estimated number of events, collected at $13 \mathrm{TeV} p p$ collisions (for $36 \mathrm{fb}^{-1}$ data sample) assuming $100 \%$ detection efficiency (last column).

is shown in Fig. 2(a). The clear peak above a background is seen in the region around $125 \mathrm{GeV}$. The ATLAS experiment observes 95 events in the mass window $118-129 \mathrm{GeV}$ with an estimated background $23.6 \pm 1.6$ events and an expected signal 54 events. The signal strength in the SM units, $\mu$, is measured to be $1.28 \pm 0.20$. The fiducial cross section measurement is performed and the extracted total cross section multiplied by the $\mathrm{BR}\left(h \rightarrow Z Z^{\star}\right)$ is $1.73 \pm 0.25 \mathrm{pb}$. It agrees with the $\mathrm{SM}$ value of $1.34 \pm 0.09 \mathrm{pb}$ within $1.4 \sigma$. Fig. 2(b) shows the values of the measured fiducial cross sections in the main production modes. No deviation from the SM is observed.

\subsection{The $h \rightarrow \gamma \gamma$ mode [21]}

The signature of the $h \rightarrow \gamma \gamma$ decay channel is two isolated photons with an invariant mass equal to $m_{h}$. To increase the discovery potential, the ATLAS experiment subdivided events into independent categories having different expected $m_{\gamma \gamma}$ resolution and signal-to-background (S/B) 


\begin{tabular}{c|c|c|c}
\hline Parameter & Value & Reference & Comment \\
\hline Mass & $125.36 \pm 0.41 \mathrm{GeV}$ & {$[13]$} & $125.09 \pm 0.24 \mathrm{GeV}$ with CMS [14] \\
Signal strength $\mu$ & $1.18 \pm 0.15$ & {$[15]$} & $1.09 \pm 0.10$ with CMS [16] \\
$\mu$ in $h \rightarrow \gamma \gamma$ mode & $1.17_{-0.26}^{+0.28}$ & {$[15]$} & $5.2 \sigma$ (discovery) \\
$\mu$ in $h \rightarrow 4 \ell$ mode & $1.46_{-0.34}^{+0.40}$ & {$[15]$} & $8.1 \sigma$ (discovery) \\
$\mu$ in $h \rightarrow W W^{\star} \rightarrow \ell v \ell v$ mode & $1.18_{-0.21}^{+0.24}$ & {$[15]$} & $6.5 \sigma$ (discovery) \\
$\mu$ in $h \rightarrow \tau \tau$ mode & $1.44_{-0.37}^{+0.42}$ & {$[15]$} & $4.5 \sigma$ (evidence) \\
$\mu$ in $h \rightarrow b \bar{b}$ mode & $0.63_{-0.37}^{+0.39}$ & {$[15]$} & $1.4 \sigma$ \\
$\mu$ in ggF production & $1.23_{-0.20}^{+0.23}$ & {$[15]$} & $1.03_{-0.15}^{+0.17}$ with CMS [16] \\
$\mu$ in VBF production & $1.23_{-0.32}^{+0.32}$ & {$[15]$} & $1.18_{-0.23}^{+0.25}$ with CMS [16] \\
$\mu$ in $V h$ production & $0.80 \pm 0.36$ & {$[15]$} & $0.84_{-0.38}^{+0.40}$ with CMS [16] \\
$\mu$ in $t$ th production & $1.81 \pm 0.80$ & {$[15]$} & $2.3_{-0.6}^{+0.7}$ with CMS [16] \\
\hline Spin/parity & $0^{+}$ & {$[17]$} & $4 \ell, \ell v \ell v, \gamma \gamma$ modes \\
Width & $\leq 22.7 \mathrm{MeV} \mathrm{(95 \%} \mathrm{CL)}$ & {$[18]$} & Off-shell $h \rightarrow W W / Z Z$ \\
BR $(h \rightarrow$ invisible $)$ & $\leq 0.28(95 \% \mathrm{CL})$ & {$[19]$} & WIMP searches \\
\hline
\end{tabular}

Table 2: Brief summary of the ATLAS results on the $h$ boson measurements at $8 \mathrm{TeV}$.

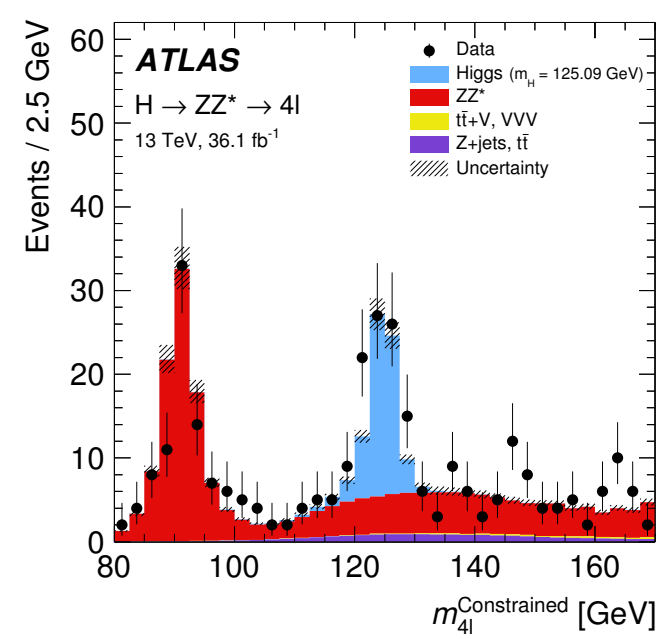

(a)

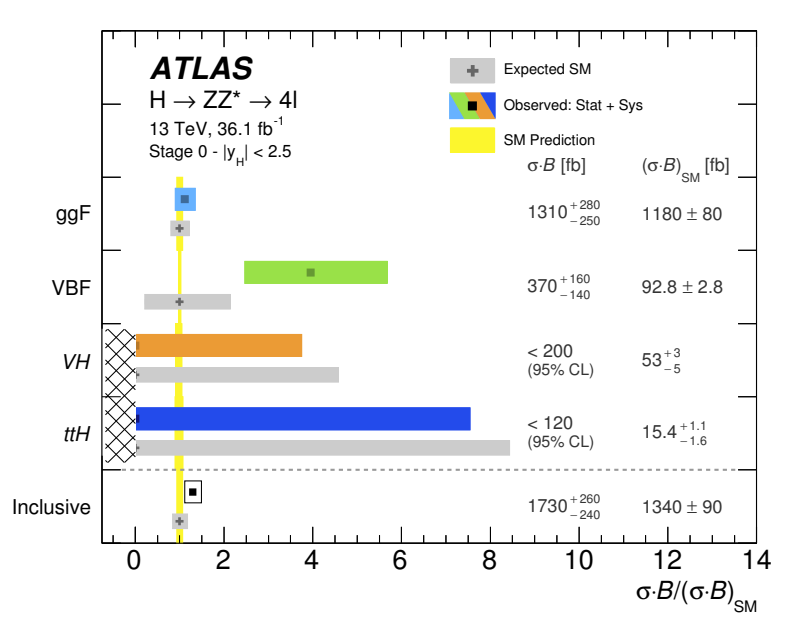

(b)

Figure 2: Results of the $h \rightarrow Z Z^{\star} \rightarrow 4 \ell$ study at $13 \mathrm{TeV}$ in the ATLAS experiment [20]. (a) $m_{4 \ell}$ distributions for the selected candidates compared to the background expectation in the mass range $80-170 \mathrm{GeV}$. The signal expectation for the $m_{h}=125 \mathrm{GeV}$ hypothesis is also shown. (b) The measured fiducial cross sections separately in the main production modes. They are normalized to the SM predictions.

ratio and optimized for the best separation of the Higgs boson production processes. The $m_{\gamma \gamma^{-}}$ distribution after the corresponding re-weighting is given in Fig. 3(a) together with the spectra after a background subtraction [21]. The excess of events with more than $5 \sigma$ significance is clearly seen around $125 \mathrm{GeV}$. The value of the signal strength in the SM units is measured to be $0.99 \pm 0.15$. Fig.3(b) shows the values of $\mu$ in the main production modes. No deviation from the SM is found. 
In addition, the fiducial cross section at $13 \mathrm{TeV}$ multiplied by $\mathrm{BR}(h \rightarrow \gamma \gamma)$, defined for the kinematic range $E_{T}^{\gamma 1} \geq 40 \mathrm{GeV}, E_{T}^{\gamma 2} \geq 30 \mathrm{GeV}$, and $|\eta|^{\gamma} \leq 2.37$, is measured to be $55 \pm 10 \mathrm{fb}$, in perfect agreement with the SM prediction of $64 \pm 2 \mathrm{fb}$.

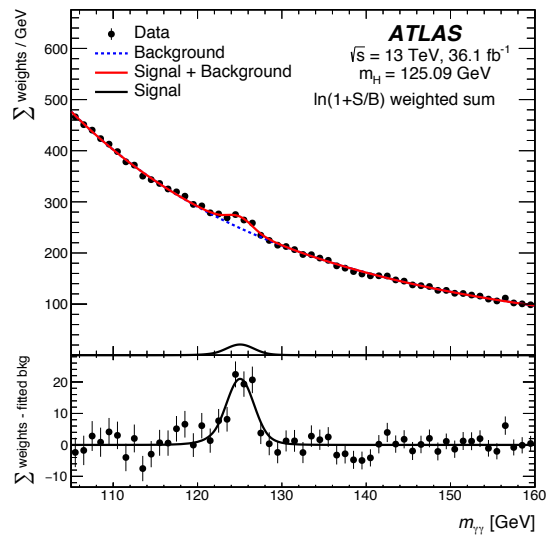

(a)

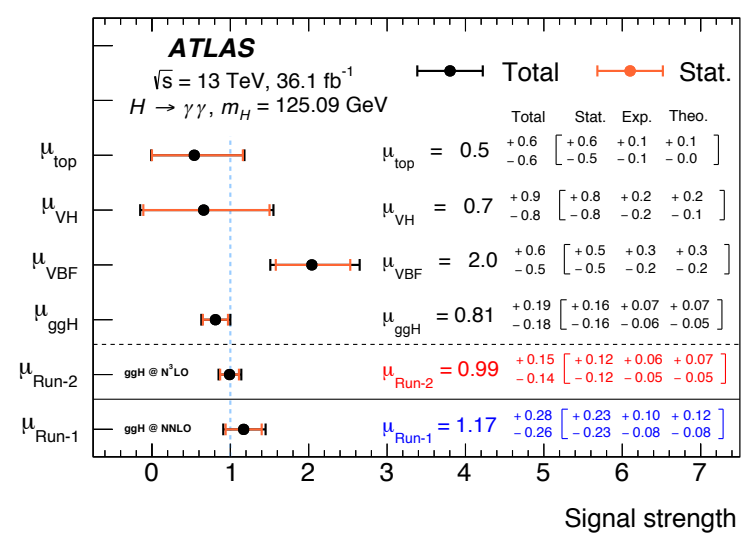

(b)

Figure 3: Results of the $h \rightarrow \gamma \gamma$ study at $13 \mathrm{TeV}$ in the ATLAS experiment [21]. (a) $m_{\gamma \gamma}$-distribution of diphoton candidates after all selections. The result of a fit to the data with the sum of a SM Higgs boson signal (with $m_{h}=125 \mathrm{GeV}$ and free signal strength) and a background is superimposed. The residuals of the data with respect to the fitted background are displayed in the lower panel. (b) The measured values of the signal strength $\mu$ in the main production modes. Also shown the values of $\mu$ measured earlier with the combined 7 and $8 \mathrm{TeV}$ data.

\subsection{A combination of the $h \rightarrow Z Z^{\star} \rightarrow 4 \ell$ and $h \rightarrow \gamma \gamma$ channels [22]}

The results obtained for the individual channels $h \rightarrow Z Z^{\star} \rightarrow 4 \ell$ and $h \rightarrow \gamma \gamma$ are combined to extract a Higgs boson signal strength in different production mechanisms (Fig. 4(a)) and its production cross section at $13 \mathrm{TeV} p p$ energy. The average signal strength is measured to be $\mu=$ $1.09 \pm 0.12$, in good agreement with the SM prediction. The measured $h$ production cross section $\sigma(p p \rightarrow h+X)=57.0_{-5.9}^{+6.0}$ (stat) ${ }_{-3.3}^{+4.0}$ (syst) pb is compatible within $1 \sigma$ with the theoretical value $55.6_{-3.4}^{+2.4} \mathrm{pb}$. It is obtained from fiducial measurements and also combined with older results at 7 and $8 \mathrm{TeV}$ (Fig.4(b)).

\subsection{The $h \rightarrow W W^{\star} \rightarrow \ell v \ell v$ mode [23]}

The signature in the $h \rightarrow W W^{\star} \rightarrow \ell v \ell v$ decay channel is two isolated opposite-sign leptons (electrons or muons) and sizeable $E_{T}^{\text {miss }}$ due to neutrinos so one cannot reconstruct the Higgs boson mass. Instead, its transverse mass reconstruction is possible. It is calculated as

$m_{T}=\sqrt{\left(E_{T}^{\ell \ell}+E_{T}^{\text {miss }}\right)^{2}-\left|\mathbf{P}_{\mathbf{T}}^{\ell \ell}+\mathbf{E}_{\mathbf{T}}^{\text {miss }}\right|^{2}}$, where $E_{T}^{\ell \ell}=\sqrt{\left|P_{T}^{\ell \ell}\right|^{2}+m_{\ell \ell}^{2}}$ and $P_{T}^{\ell \ell}\left(m_{\ell \ell}\right)$ are transverse momentum (invariant-mass) of the di-lepton system, respectively. So knowledge of a background normalization and shapes is of importance. The event sample is subdivided in accordance with the number of jets $(0,1$ or $\geq 2)$. For the 0 and 1 jet cases the ggF mechanism is expected to be dominant while for $\geq 2$ jets case signal events are mostly due to the VBF mechanism. Only the $e \mu$ 


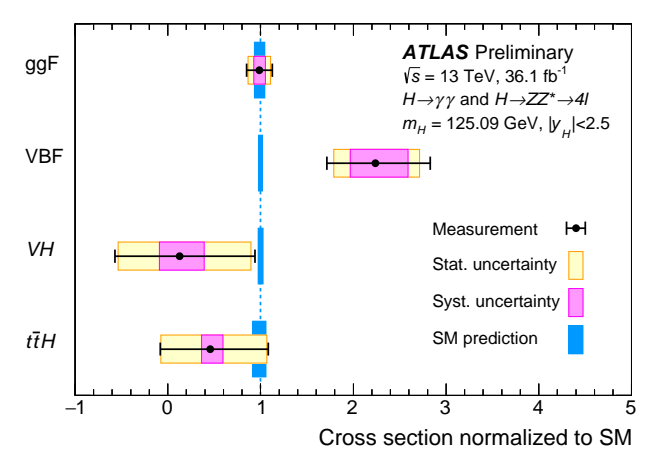

(a)

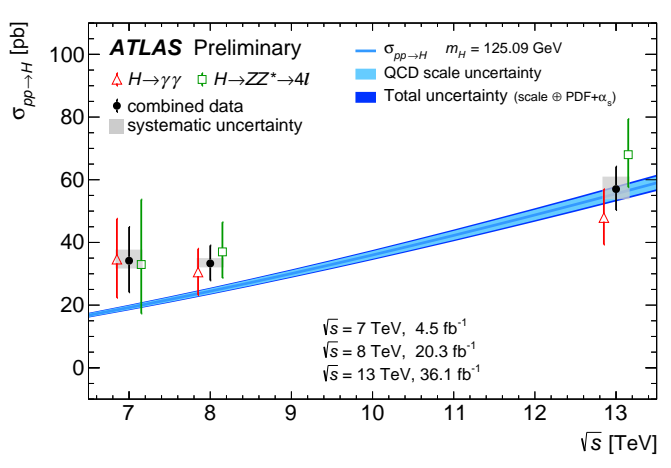

(b)

Figure 4: Results of the preliminary $h \rightarrow Z Z^{\star} \rightarrow 4 \ell$ and $h \rightarrow \gamma \gamma$ combination at $13 \mathrm{TeV}$ in the ATLAS experiment [22]. (a) The measured values of the signal strength in the main production modes. (b) The measured total $h$ boson production cross sections at 7,8 and $13 \mathrm{TeV}$ together with theoretical predictions.

final state is considered. The $m_{T}$-distribution before and after a background subtraction is shown in Fig.5(a). A clear excess of events is seen in the region $80-140 \mathrm{GeV}$. The observed (expected) signal significance at $125 \mathrm{GeV}$ for the $\mathrm{ggF} h$ production is $6.3 \sigma(5.2 \sigma)$, respectively. The corresponding signal strength in the SM units is $1.21 \pm 0.22$, and the ggF cross section multiplied by $\mathrm{BR}\left(h \rightarrow W W^{\star}\right)$ is measured to be $12.6 \pm 2.2 \mathrm{pb}$, in perfect agreement with the theoretical prediction $10.4 \pm 0.6 \mathrm{pb}$. The observed (expected) signal significance in the VBF production mode is $1.9 \sigma$ ( $2.7 \sigma), \mu=0.62 \pm 0.37$ and the measured cross section is $0.50 \pm 0.30 \mathrm{pb}$ being compatible with the expected value of $0.81 \pm 0.02 \mathrm{pb}$. The correlation between the measured $\mathrm{ggF}$ and VBF cross sections is shown in Fig.5(b).

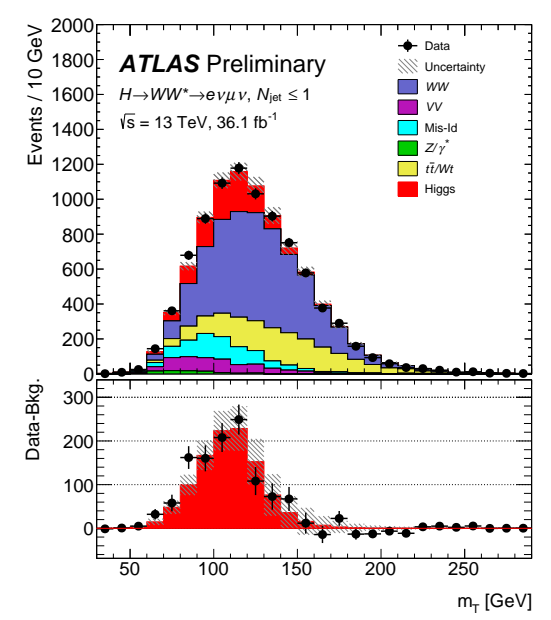

(a)

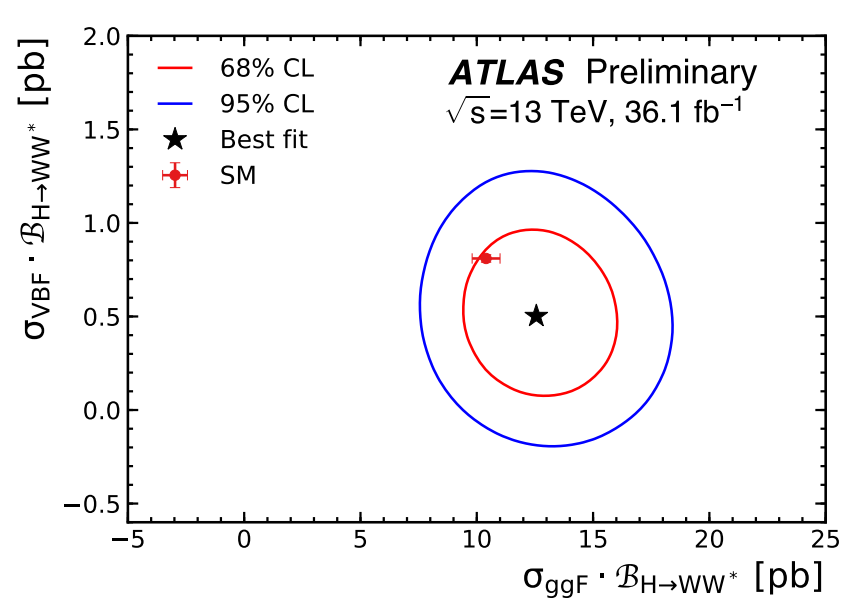

(b)

Figure 5: Results of the $h \rightarrow W W^{\star} \rightarrow \ell v \ell v$ study at $13 \mathrm{TeV}$ in the ATLAS experiment [23]. (a) $m_{T}$ distribution before and after a background subtraction in the case of less than two jets in an event. (b) The correlation between the measured ggF and VBF cross sections. 


\section{The $V h \rightarrow b \bar{b}+X$ decay channel [24]}

The $V h \rightarrow b \bar{b}+X$ signature includes two jets originating from $b$-quarks with an invariant mass close to $m_{h}$. In addition, only events with tight lepton(s) and/or high $E_{T}^{\text {miss }}$ depending on the $Z / W$ final state: $Z \rightarrow v v$ (0 leptons), $W \rightarrow \ell v$ (one lepton) and $Z \rightarrow \ell \ell$ (two leptons) are considered. A multivariate analysis is performed to discriminate the signal from a background. The procedure is successfully tested on the $(W / Z) Z$ process with subsequent $Z \rightarrow b \bar{b}$ decay. The observed (expected) signal significance is $3.5 \sigma(3.0 \sigma)$. This corresponds to the signal strength $\mu=1.20_{-0.36}^{+0.42}$ at $13 \mathrm{TeV}$. Fig.6(a) shows the measured $\mu$ separately in the $W h$ and $Z h$ production modes. The results after the combination with the 7 and $8 \mathrm{TeV}$ data are given in Fig.6(b). The combined observed (expected) significance is $3.6 \sigma(4.0 \sigma)$; the signal strength is measured to be $0.90 \pm 0.27$, in agreement with the SM prediction.

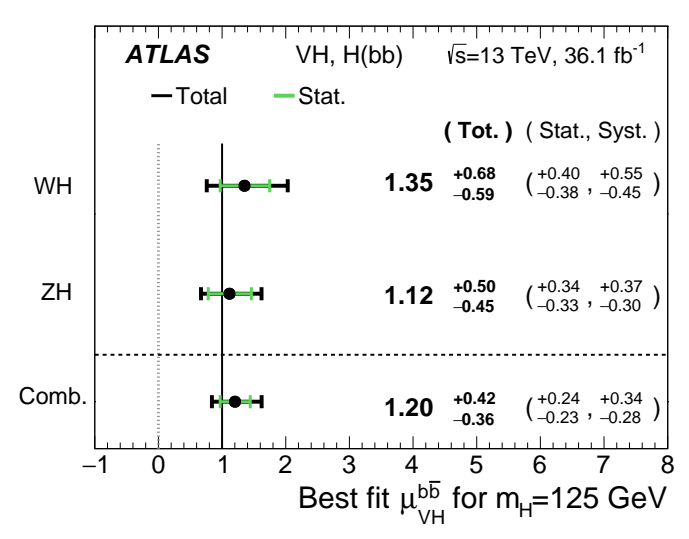

(a)

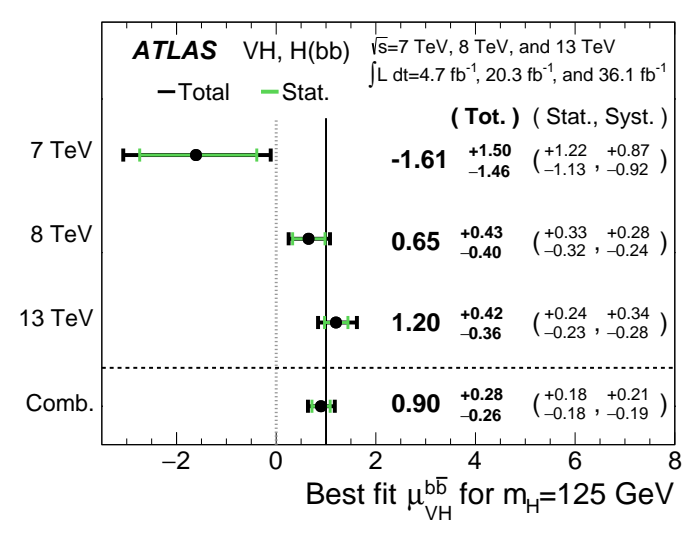

(b)

Figure 6: The observed signal strength $\mu$ in the $V h \rightarrow b \bar{b}+X$ production mode in the ATLAS experiment [24]. (a) $13 \mathrm{TeV}$ data, separately for the $W h$ and $Z h$ production modes and combined result. (b) 7, 8 and 13 $\mathrm{TeV}$ data as well as their combination.

\section{The $t \bar{t} h$-production [25]}

The $t \bar{t} h$-production mechanism is studied using a variety of multi-lepton final states. A multivariate technique to discriminate the signal from a background is used. The measured $\mu$ is $1.6_{-0.4}^{+0.5}$ (Fig.7(a)) which corresponds to observed (expected) signal significance $4.1 \sigma(2.8 \sigma)$. After the combination with another signatures (Fig.7(b)) the measured $\mu$ is found to be $1.2 \pm 0.3$ [25]. The observed (expected) signal significance is $4.2 \sigma(3.8 \sigma)$.

\section{Search for the Higgs boson beyond the $\mathrm{SM}$ at $13 \mathrm{TeV}$}

The simplest model is the so-called Narrow Width Approximation (NWA) where additional high-mass Higgs boson $(H)$ behaves as the SM Higgs boson $h$, except the width is fixed to be equal to $4 \mathrm{MeV}$, which is the expected width of the SM Higgs boson at $m_{h}=125 \mathrm{GeV}$. This 


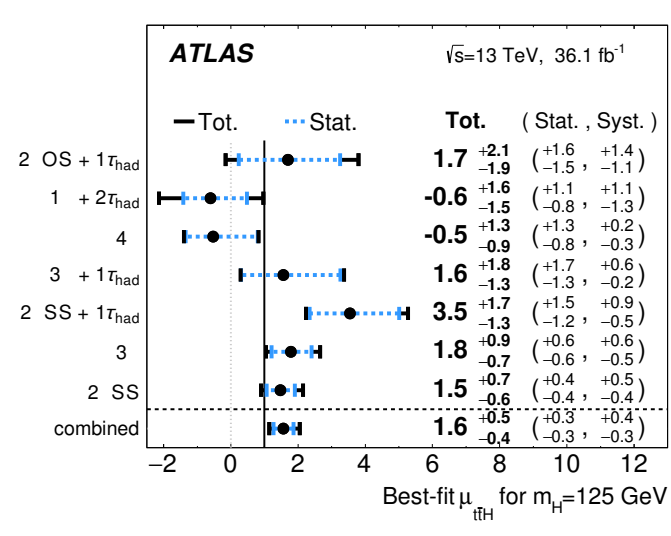

(a)

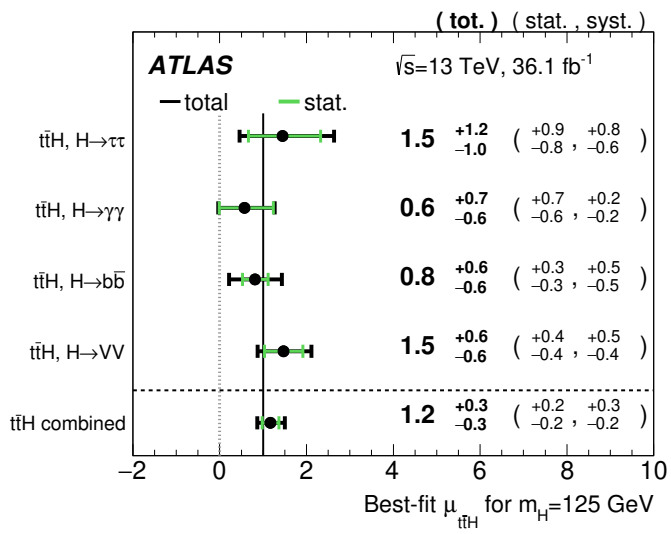

(b)

Figure 7: The observed signal strength in the $t \bar{t} h$ production mode at $13 \mathrm{TeV}$ in the ATLAS experiment [25]. (a) Multi-lepton final state. (b) All final states.

simplified model allows to produce MC samples easily. In addition, the interference of the signal with background processes can be neglected simplifying the analysis. The predictions for the Higgs boson production cross section at $13 \mathrm{TeV}$ in different mechanisms are shown in Fig.8(a) as function of $m_{H}$. It is interesting to note that above $1.5 \mathrm{TeV}$ mass the VBF mechanism starts to dominate. Another models contain five Higgs bosons (neutral light and neutral heavy CP-even states, $h$ and $H$, one CP-odd neutral state $A$ and two charged states, $H^{+}$and $H$ ). Four masses $m_{h}, m_{H}, m_{A}, m_{H^{+}}$, mixing angle $\alpha$ between the light and the heavy neutral Higgs boson, and the ratio $\tan \beta$ of two vacuum expectation values are usual free parameters.

In this note only ATLAS results on heavy neutral scalar Higgs boson searches in some bosonic decay modes are covered. The interpretation is performed in the NWA framework.

Table 3 shows expected $H$ branching ratios at $m_{H}=1 \mathrm{TeV}$ as well as estimated number of events, collected at $7 \mathrm{TeV}, 8 \mathrm{TeV}$ and $13 \mathrm{TeV}$ pp collisions (for $5 \mathrm{fb}^{-1}, 20 \mathrm{fb}^{-1}$ and $36 \mathrm{fb}^{-1}$ data samples, respectively) assuming $100 \%$ detection efficiency. It is seen that for some of the channels considered the rates are too small at 7 and $8 \mathrm{TeV}$ while they are rather big at $13 \mathrm{TeV}$.

\begin{tabular}{c|c|c|c|c}
\hline Decay mode & BR, \% & Rates at $7 \mathrm{TeV}$ & Rates at $8 \mathrm{TeV}$ & Rates at $13 \mathrm{TeV}$ \\
\hline$H \rightarrow W W \rightarrow \ell v \ell v$ & $\approx 2.7 \%$ & $\approx 2$ & $\approx 30$ & $\approx 500$ \\
$H \rightarrow W W \rightarrow \ell v q \bar{q}$ & $\approx 17 \%$ & $\approx 10$ & $\approx 200$ & $\approx 4000$ \\
$H \rightarrow Z Z \rightarrow 4 \ell$ & $\approx 0.17 \%$ & $\approx 0.15$ & $\approx 2$ & $\approx 35$ \\
$H \rightarrow Z Z \rightarrow \ell \ell v v$ & $\approx 1.0 \%$ & $\approx 0.9$ & $\approx 12$ & $\approx 200$ \\
$H \rightarrow Z Z \rightarrow \ell \ell q \bar{q}$ & $\approx 2.9 \%$ & $\approx 2.6$ & $\approx 35$ & $\approx 500$ \\
$H \rightarrow Z Z \rightarrow v v q \bar{q}$ & $\approx 8.5 \%$ & $\approx 7.5$ & $\approx 100$ & $\approx 1700$ \\
\hline
\end{tabular}

Table 3: Expected $H$ branching ratios at $m_{H}=1 \mathrm{TeV}$ as well as estimated number of events, collected at 7 $\mathrm{TeV}, 8 \mathrm{TeV}$ and $13 \mathrm{TeV} p p$ collisions (for $5 \mathrm{fb}^{-1}, 20 \mathrm{fb}^{-1}$ and $36 \mathrm{fb}^{-1}$ data samples, respectively) assuming $100 \%$ detection efficiency (last three columns). 


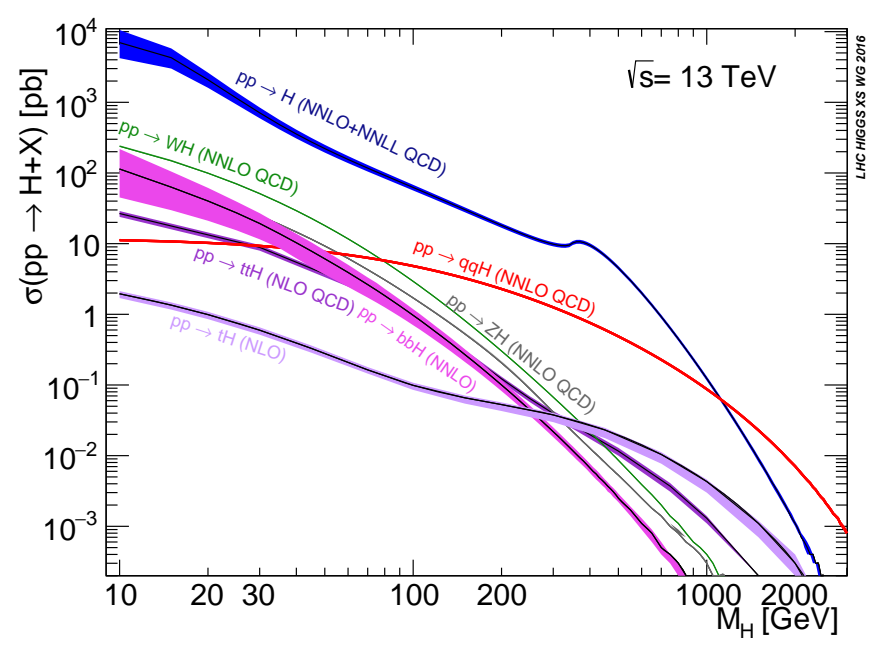

(a)

Figure 8: Predicted BSM Higgs boson production cross section at $13 \mathrm{TeV}$ in the NWA approach as function of $m_{H}$ [12].

\subsection{The $H \rightarrow \gamma \gamma$ mode [26]}

The $H \rightarrow \gamma \gamma$ decay mode has a special interest because of the observation of an excess at around $750 \mathrm{GeV}$ in the di-photon invariant-mass distribution both by the ATLAS and CMS experiments in the 2015 data sample [27]-[28]. In the ATLAS case, the deviation from the SM was at $3.4 \sigma$ level (local significance). The ATLAS Collaboration performed a new analysis based on ten times larger dataset mostly obtained in 2016 . The search mass range is $200-2700 \mathrm{GeV}$. Some 32755 events with hard photons were selected and the resulting $m_{\gamma \gamma}$-distribution is shown in Fig. 9(a). No significant excess is observed. The resulting 95\% CL upper limit on the fiducial cross section multiplied by the $\mathrm{BR}(H \rightarrow \gamma \gamma)$ is given in Fig. 9(b).

\subsection{The $H \rightarrow W W \rightarrow \ell v \ell v$ mode [29]}

The mass range between $300-3000 \mathrm{GeV}$ is covered by the $H \rightarrow W W \rightarrow e v \mu \nu$ decay channel. Selection criteria are specially optimized for the high-mass case and a multivariate analysis is performed. Events are subdivided into ggF- and two VBF-enriched categories. No significant excess above a background is found in the search mass range (Fig. 10(a)-Fig. 10(b)). 95\% CL upper limits on $\sigma_{H} \times \mathrm{BR}(H \rightarrow W W)$ are established separately in the ggF and VBF production mechanisms in the framework of the NWA model. They are $6.4 \mathrm{pb}(8 \mathrm{fb})$ at $m_{H}=200 \mathrm{GeV}\left(m_{H}=\right.$ $4000 \mathrm{GeV})$ in the $\mathrm{ggF}$ case and $1.3 \mathrm{pb}(6 \mathrm{fb})$ at $m_{H}=300 \mathrm{GeV}\left(m_{H}=3000 \mathrm{GeV}\right)$ in the VBF case.

\section{Conclusion and short-term plans}

In conclusion: 


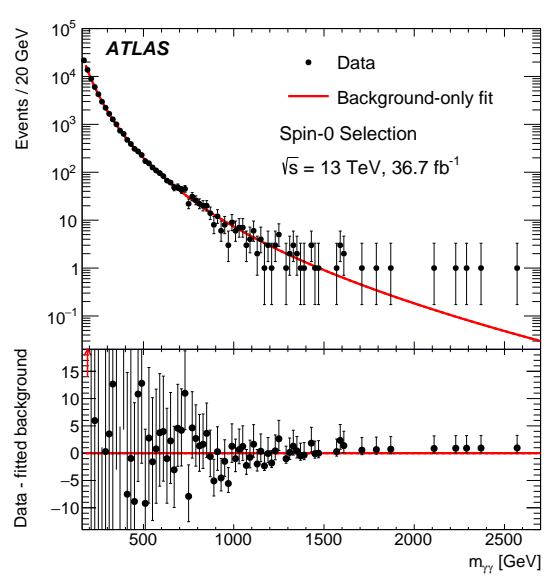

(a)

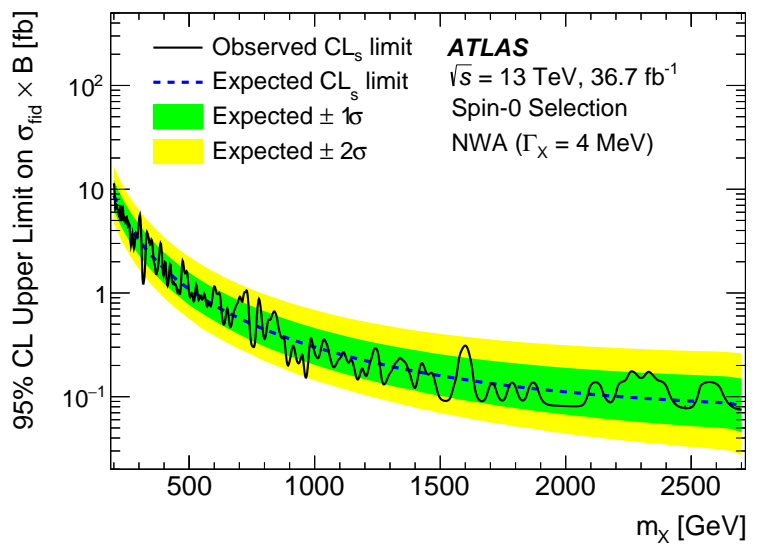

(b)

Figure 9: Results of the $H \rightarrow \gamma \gamma$ study at $13 \mathrm{TeV}$ in the ATLAS experiment [26]. (a) $m_{\gamma \gamma}$-distribution of diphoton candidates after all selections. (b) $95 \%$ CL upper limit on the fiducial cross section multiplied by the $\operatorname{BR}(H \rightarrow \gamma \gamma)$. as a function of $m_{H}$.

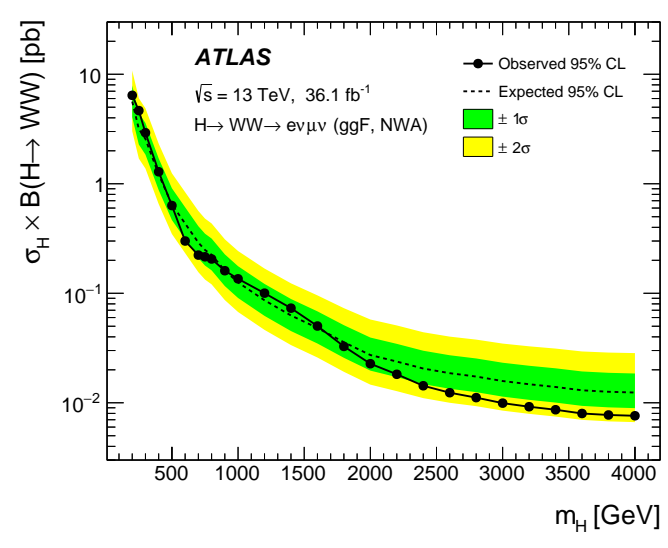

(a)

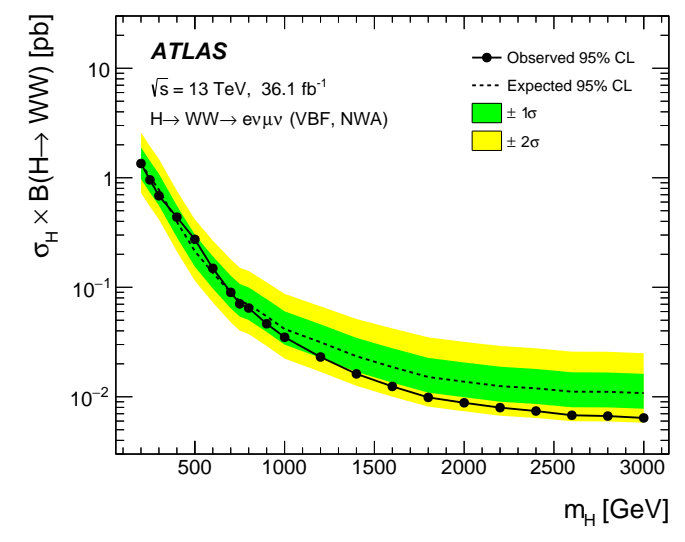

(b)

Figure 10: Results of the $H \rightarrow W W \rightarrow \ell v \ell v$ study at $13 \mathrm{TeV}$ in the ATLAS experiment [29]: 95\% CL upper limits on the cross section multiplied by the $\mathrm{BR}(H \rightarrow W W)$ as a function of $m_{H}$ in two production modes. (a) ggF production mode. (b) VBF production mode.

- With 7, 8 and $13 \mathrm{TeV}$ LHC data, the ATLAS Collaboration studied a lot of SM processes and measured their cross sections with high precision. All of them are in agreement with recent theoretical calculations;

- Using the same datasets, Higgs boson properties such as its couplings, mass, spin and parity were investigated. No deviation from the SM is found;

- With $36 \mathrm{fb}^{-1}$ of $13 \mathrm{TeV}$ LHC data, we obtained preliminary results reconfirming SM-like Higgs boson discovery in the $4 \ell$ and $\gamma \gamma$ modes making evidence for the $h \rightarrow b \bar{b}$ decay and 
$t \bar{t} h$-production;

- The ATLAS Collaboration also performed searches for new physics in many final states. Nothing was found yet. Strict limits on production cross sections of new particles were put;

- We plan to continue to improve existing measurements and to search for deviations from the SM with new 13-14 TeV data.

The short-term LHC and ATLAS (CMS) perspectives are the following:

- In 2018, it is expected to collect the data sample in $p p$ collisions at $13 \mathrm{TeV}$ which corresponds to 55-60 $\mathrm{fb}^{-1}$ integrated luminosity, so the complete ATLAS and CMS datasets will be of $\approx$ $150 \mathrm{fb}^{-1}$. A lot of papers based on these samples are planned to be published;

- The LHC as well as the ATLAS and CMS experiments will be upgraded during 2019-2020 shutdown to operate at luminosities up to $3 \times 10^{34} \mathrm{~cm}^{-2} \mathrm{~s}^{-1}$;

- In 2021-2023 the run at increased energy (from $13 \mathrm{TeV}$ to $14 \mathrm{TeV}$ ) is foreseen aiming to collect $300 \mathrm{fb}^{-1}$. More precise measurements of the $h$ coupling and its main decay channels will be made, a discovery of new physics or strict upper limit settings on the cross section of new particles are expected. In addition, studies of rare $B$-meson decays and detailed investigations of ion-ion and proton-ion collisions will be continued;

- The next upgrade of the LHC and the experiments ${ }^{3}$ will be performed during 2024-2026 shutdown. After this shutdown the LHC develops into the High Luminosity stage (HLLHC) and the general-purpose experiments should be able to work up at $7 \times 10^{34} \mathrm{~cm}^{-2} \mathrm{~s}^{-1}$ luminosity. The total integrated luminosity foreseen for the HL-LHC phase is $3000 \mathrm{fb}^{-1}$.

\section{References}

[1] L. Evans and P. Bryant (editors), LHC Machine, JINST 3 (2008) S08001.

[2] ATLAS Collaboration, The ATLAS Experiment at the CERN Large Hadron Collider, JINST 3 (2008) S08003.

[3] CMS Collaboration, The CMS Experiment at the CERN Large Hadron Collider, JINST 3 (2008) S08002.

[4] LHCb Collaboration, The LHCb Experiment at the CERN Large Hadron Collider, JINST 3 (2008) S08005.

[5] ALICE Collaboration, The ALICE Experiment at the CERN Large Hadron Collider, JINST 3 (2008) S08004.

[6] ATLAS Collaboration, Observation of a new particle in the search for the Standard Model Higgs boson with the ATLAS detector at the LHC, Phys. Lett. B 716 (2012) 1, [arXiv: 1207. 7214]

[7] CMS Collaboration, Observation of a new boson at a mass of $125 \mathrm{GeV}$ with the CMS experiment at the LHC, Phys. Lett. B 716 (2012) 30, [arXiv:1207. 7235]

\footnotetext{
${ }^{3}$ The LHCb upgrade is planned to 2030.
} 
[8] ATLAS Collaboration, Standard Model Total Cross Section Measurements, directory https://atlas.web.cern.ch/Atlas/GROUPS/PHYSICS/CombinedSummaryPlots/SM

[9] R. Barate et al., Search for the standard model Higgs boson at LEP, Phys. lett. B 565 (2003) 61, [hep-ex/0306033]

[10] Plot $\Delta \chi^{2}$ vs $m_{h}$ at http://lepewwg.web.cern.ch/LEPEWWG/

[11] CDF and D0 Collaborations, Evidence for a particle produced in association with weak bosons and decaying to a bottom-antibottom quark pair in Higgs boson searches at the Tevatron, Phys. Rev. Lett. 109 (2012) 071804.

[12] LHC Higgs Cross Section Working Group, D. de Florian et al, 4. Deciphering the Nature of the Higgs Sector [arXiv:1610.07922]

[13] ATLAS Collaboration, Measurement of the Higgs boson mass from the $h \rightarrow \gamma \gamma$ and $h \rightarrow Z Z^{\star} \rightarrow 4 \ell$ channels in pp collisions at center-of-mass energies of 7 and $8 \mathrm{TeV}$ with the ATLAS detector, Phys. Rev. D 90 (2014) 052004, [arXiv: 1406.3827 ]

[14] ATLAS and CMS Collaborations, Combined Measurement of the Higgs Boson Mass in pp Collisions at 7 and 8 TeV with the ATLAS and CMS Experiments, Phys. Rev. Lett. 114 (2015) 191803, [arXiv:1503.07589]

[15] ATLAS Collaboration, Measurements of the Higgs boson production and decay rates and coupling strengths using pp collision data at 7 and $8 \mathrm{TeV}$ in the ATLAS experiment, Eur. Phys. Journal C 76 (2016) 6, [arXiv:1507.04548]

[16] ATLAS and CMS Collaborations, Measurements of the Higgs boson production and decay rates and constraints on its couplings from a combined ATLAS and CMS analysis of the LHC pp collision data at 7 and 8 TeV, Journal of High Energy Phys. 08 (2016) 045, [arXiv: 1606.02266 ]

[17] ATLAS Collaboration, Study of the spin and parity of the Higgs boson in diboson decays with the ATLAS detector, Eur. Phys. Journal C 75 (2015) 476, [arXiv: 1506.05669 ]

[18] ATLAS Collaboration, Constraints on the off-shell Higgs boson signal strength in the high-mass ZZ and WW final states with the ATLAS detector, Eur. Phys. Journal C 75 (2015) 335,

[arXiv:1503.01060]

[19] ATLAS Collaboration, Search for invisible decays of a Higgs boson using vector-boson fusion in pp collisions at $8 \mathrm{TeV}$ with the ATLAS detector, Journal High Energy Phys. 01 (2016) 172, [arXiv:1508.07869]

[20] ATLAS Collaboration, Measurement of the Higgs boson coupling properties in the $h \rightarrow Z Z^{\star} \rightarrow 4 \ell$ decay channel at $13 \mathrm{TeV}$ with the ATLAS detector, JHEP 03 (2018) 095, [arXiv: 1712.02304 ]

[21] ATLAS Collaboration, Measurements of Higgs boson properties in the diphoton decay channel with $36 \mathrm{fb}^{-1}$ of pp collision data at $13 \mathrm{TeV}$ with the ATLAS detector, submitted to Phys. Rev. D, [arXiv:1802.04146]

[22] ATLAS Collaboration, Combined measurements of Higgs boson production and decay in the $h \rightarrow Z Z^{\star} \rightarrow 4 \ell$ and $h \rightarrow \gamma \gamma$ channels using $13 \mathrm{TeV}$ pp collision data collected with the ATLAS experiment, ATLAS-CONF-2017-047, https://cds.cern.ch/record/2273854

[23] ATLAS Collaboration, Measurement of gluon fusion and vector boson fusion Higgs boson production cross-sections in the $h \rightarrow W W^{\star} \rightarrow \ell v \ell v$ decay channel in pp collisions at $13 \mathrm{TeV}$ with the ATLAS detector, ATLAS-CONF-2018-004, http://cds.cern.ch/record/2308392 
[24] ATLAS Collaboration, Evidence for the $h \rightarrow b \bar{b}$ decay with the ATLAS detector, JHEP 12 (2017) 24, [arXiv:1708.03299]

[25] ATLAS Collaboration, Evidence for the associated production of the Higgs boson and a top quark pair with the ATLAS detector, Phys. Rev. D 97 (2018) 072003, [arXiv:1712.08891]

[26] ATLAS Collaboration, Search for new phenomena in high-mass diphoton final states using $37 \mathrm{fb}^{1}$ of proton-proton collisions collected at $13 \mathrm{TeV}$ with the ATLAS detector, Phys. Lett. B 775 (2017) 105, [arXiv:1707.04147]

[27] ATLAS Collaboration, Search for resonances in diphoton events at $13 \mathrm{TeV}$ with the ATLAS detector, JHEP 09 (2016) 1, [arXiv: 1606.03833 ]

[28] CMS Collaboration, Search for high-mass diphoton resonances in proton-proton collisions at $13 \mathrm{TeV}$ and combination with 8 TeV search, Phys. Lett. B 767 (2017) 147, [arXiv: 1609.02507 ].

[29] ATLAS Collaboration, Search for heavy resonances decaying into $W W$ in the ev $\mu v$ final state in $p p$ collisions at $13 \mathrm{TeV}$ with the ATLAS detector, Europ. Phys. Journal C 78 (2018) 24,

[arXiv:1710.01123].

\section{DISCUSSION}

JIULIO AURIEMMA: Could you please comment about statistical significance of the Higgs boson signal, for example, given in your slide 14 ?

ILYA TSUKERMAN: We checked two hypotheses:

Hypothesis 1: the observed excess of events above the background is due to the Standard Model Higgs boson;

Hypothesis 2: the observed excess is due to occasional background fluctuation.

The number of standard deviations $(\sigma)$ given in the last column of Table in slide 14 corresponds to the hypothesis 2 while the measured signal strength in the SM units (second column in the mentioned table) corresponds to the hypothesis 1. You see that the quoted global signal strength of $1.18 \pm 0.15$ is almost within one $\sigma$ compatible with unity while it is incompatible with zero (more than $10 \sigma$ deviation). This means that the observed particle is a Higgs boson.

QUESTION: What are perspectives of new physics searches at the LHC?

ILYA TSUKERMAN: New physics is not yet observed in the LHC experiments. In backup slides 71-72 which I am showing now you see summaries of ATLAS lower mass limits on different SUSY-predicted and other exotic particles and objects. If nothing will be observed, we simply put more strict limits analysing more and more experimental data. 